International Journal of Pure and Applied Mathematics

Volume 114 No. 2 2017, 329-341

ISSN: 1311-8080 (printed version); ISSN: 1314-3395 (on-line version)

url: http://www.ijpam.eu

doi: 10.12732 /ijpam.v114i2.14

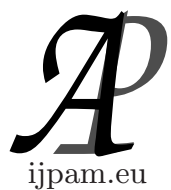

\title{
EFFECT OF STRESS JUMP ON FLOW PAST A POROUS SPHERICAL SHELL OF VARYING PERMEABILITY WITH SOLID CORE
}

\author{
Vineet Kumar Verma ${ }^{1 \S}$, Amit Kumar Gupta ${ }^{2}$ \\ ${ }^{1,2}$ Department of Mathematics and Astronomy \\ University of Lucknow \\ Lucknow, 226007, INDIA
}

\begin{abstract}
In this paper we have analytically investigated the effects of stress jump coefficient on flow past a porous spherical shell of varying permeability with solid core for steady, viscous and incompressible fluid using the Brinkman model. We have assumed that the flow is of uniform velocity far away from the sphere. Exact mathematical expressions for the velocity of fluid within and outside porous shell, streamlines of the flow and drag on the shell are obtained and exhibited graphically. The effect of stress jump coefficient and permeability parameter on the velocity, streamlines and on the drag force are investigated.
\end{abstract}

AMS Subject Classification: 76Dxx, 76Sxx

Key Words: Brinkman flow, variable permeability, porous shell, stokes flow

\section{Introduction}

The fluid flow past a porous spherical shell is an important topic in the field of flow through porous media. It is very useful in Geological engineering, Petroleum reservoirs, filtration process etc. Many authors have studied flow past spherical particle. An arbitrary Stokes flow past a porous sphere using Brinkman equation was studied by Padmawathi et. al.(1993) using continu-

Received: $\quad$ February 17, 2017

Revised: $\quad$ April 13, 2017

Published: $\quad$ May 6, 2017

(c) 2017 Academic Publications, Ltd. url: www.acadpubl.eu

$\S_{\text {Correspondence author }}$ 
ity of velocity components as well as stress components. Ochao-Tapia and Whitaker (1998) suggested a stress jump boundary condition at the interface of fluid and porous medium while discussing flow of a homogeneous fluid parallel to a porous medium. Kuznetsov (1998) used this stress jump boundary condition at the interface between a porous medium and a clear fluid to discuss flow in channels partially filled with porous medium. Kim and yuan (2005) studied the hydrodynamic properties of aggregate by modelling it as a porous spherical particle of continuous radially increasing permeability. They used Brinkman equation for the flow within the porous aggregate and continuity of the tangential and normal velocities and stresses at the interfacial conditions. Creeping flow past a solid sphere with a porous shell has been solved by J.H. Masliyah et.al.(1987) using the Stoke's and Brinkman equation. An analytical investigation for a two dimensional steady, viscous and incompressible flow past a permeable sphere embedded in another porous medium is studied by T. Grosan et al.(2010) using Brinkman model assuming a uniform shear flow far away from the sphere. Verma and Datta(2012) have studied the slow flow past a heterogeneous porous sphere with radial variation of permeability. Jones(1973), Higdon and Kojima(1981), Bhatt and Sacheti(1994), Berman(1996)and Qin and Kaloni(1993) also studied the problems related to flow past a porous spherical shell.

In the present paper we have considered the axi-symmetric flow of a viscous, incompressible fluid past a solid sphere having a porous shell of radially varying permeability $k=k_{0} r^{2}$ with stress jump condition at the interface of clear fluid and porous medium given by Ochao-Tapia and Whitaker(1995). Here $k_{0}$ is the characteristic permeability of sphere which may be taken as mean permeability, $r$ is radial distance from the centre of sphere. We use Stoke's equation to govern the flow in the free flow region and Brinkman equation to govern the flow in the porous region. As boundary conditions continuity of the velocity, normal stress and jump in tangential shear stress at the interface of porous sphere and homogeneous fluid are employed. The effect of stress jump coefficient on the flow velocity, streamlines and the drag force are investigated.

\section{Mathematical Formulation}

Consider the steady flow of a viscous and incompressible fluid past a solid sphere having a porous shell with varying permeability of thickness $(b-a)$ and the radius of solid sphere is $a$. The fluid has uniform velocity $U$ far away from the spherical structure. We assume the flow is axis symmetric and divide the flow 
field in to two regions region I and region II are outside and inside the spherical structure. The governing equation for flow in region I is Navier-Stokes equation

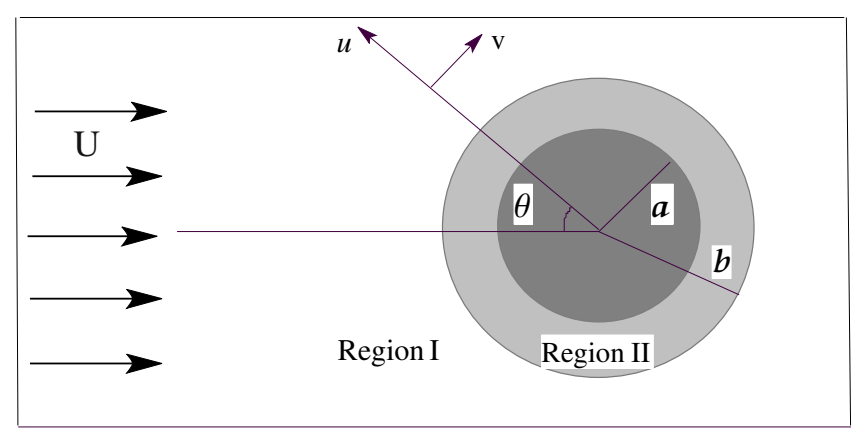

fig. 1: Sketch of the problem.

and equation of continuity i.e.

$$
\begin{gathered}
\nabla \bar{p}_{1}=\mu \nabla^{2} \bar{q}_{1} \\
\nabla \cdot \bar{q}_{1}=0
\end{gathered}
$$

where $\bar{q}_{1}$ and $\bar{p}_{1}$ are velocity and pressure in the region- I and $\mu$ is fluid viscosity. The flow inside region-II is governed by Brinkman equation and the equation of continuity i.e.

$$
\begin{gathered}
\nabla \bar{p}_{2}=-\frac{\mu}{k} \bar{q}_{2}+\mu_{e} \nabla^{2} \bar{q}_{2} \\
\nabla \cdot \bar{q}_{2}=0
\end{gathered}
$$

where $\bar{q}_{2}$ is fluid velocity and $\bar{p}_{2}$ is the pressure in the flow region II. $k(r)$ is the permeability of the porous medium which is varying radially according to the law $k=k_{0} r^{2}$, where $k_{0}$ is the characteristic permeability and $\mu_{e}$ is the effective viscosity in the porous medium. According to Nield and Bejan (2006) $\mu_{e}=\mu$ for high porosity. With these consideration Brinkman eqution (3) becomes

$$
\nabla \bar{p}_{2}=-\frac{\mu}{k} \bar{q}_{2}+\mu \nabla^{2} \bar{q}_{2}
$$

We use spherical polar coordinate system $(r, \theta, \Phi)$ with centre of sphere as origin and the line $\theta=0$ as the axis of symmetry in the direction of free stream velocity $\mathrm{U}$ as shown in fig.1. Due to axis symmetric flow all the physical quantities are 
independent of $\Phi$ thus we have $\frac{\partial}{\partial \Phi}=0$. It is convenient to use the following dimensionless variables-

$$
r=\frac{\bar{r}}{a}, u_{i}=\frac{\bar{u}_{i}}{U}, v_{i}=\frac{\bar{v}_{i}}{U}, p_{i}=a \frac{\bar{p}_{i}}{\mu U}
$$

where $u_{i}$ and $v_{i}$ are radial and tangential component of $\bar{q}_{i}$. Here index $i=1$, 2 is related to region I and II respectively. Now we can write equation(2) and (4) with these dimensionless variables as:

$$
\frac{\partial}{\partial r}\left(r^{2} u_{i}\right)+\frac{r}{\sin \theta} \frac{\partial}{\partial \theta}\left(v_{i} \sin \theta\right)=0
$$

equation (1)can be written as

$$
\begin{gathered}
\frac{\partial p_{1}}{\partial r}=\frac{\partial^{2} u_{1}}{\partial r^{2}}+\frac{2}{r} \frac{\partial u_{1}}{\partial r}+\frac{1}{r^{2}} \frac{\partial^{2} u_{1}}{\partial \theta^{2}}+\frac{\cot \theta}{r^{2}} \frac{\partial u_{1}}{\partial \theta}-\frac{2 u_{1}}{r^{2}}-\frac{2}{r^{2}} \frac{\partial v_{1}}{\partial \theta}-\frac{2 v_{1} \cot \theta}{r^{2}} \\
\frac{1}{r} \frac{\partial p_{1}}{\partial \theta}=\frac{\partial^{2} v_{1}}{\partial r^{2}}+\frac{2}{r} \frac{\partial v_{1}}{\partial r}+\frac{1}{r^{2}} \frac{\partial^{2} v_{1}}{\partial \theta^{2}}+\frac{\cot \theta}{r^{2}} \frac{\partial v_{1}}{\partial \theta}+\frac{2}{r^{2}} \frac{\partial u_{1}}{\partial \theta}-\frac{v_{1} \cos e c^{2} \theta}{r^{2}}
\end{gathered}
$$

and equation (5) can be written as

$$
\begin{aligned}
& -\frac{\partial p_{2}}{\partial r}=\frac{a^{2}}{k(r)} u_{2}-\left(\frac{\partial^{2} u_{2}}{\partial r^{2}}+\frac{2}{r} \frac{\partial u_{2}}{\partial r}+\frac{1}{r^{2}} \frac{\partial^{2} u_{2}}{\partial \theta^{2}}+\frac{\cot \theta}{r^{2}} \frac{\partial u_{2}}{\partial \theta}-\frac{2 u_{2}}{r^{2}}\right. \\
& \left.-\frac{2}{r^{2}} \frac{\partial v_{2}}{\partial \theta}-\frac{2 v_{2} \cot \theta}{r^{2}}\right) \\
& -\frac{1}{r} \frac{\partial p_{2}}{\partial \theta}=\frac{a^{2}}{k(r)} v_{2}-\left(\frac{\partial^{2} v_{2}}{\partial r^{2}}+\frac{2}{r} \frac{\partial v_{2}}{\partial r}+\frac{1}{r^{2}} \frac{\partial^{2} v_{2}}{\partial \theta^{2}}+\frac{\cot \theta}{r^{2}} \frac{\partial v_{2}}{\partial \theta}\right. \\
& \left.+\frac{2}{r^{2}} \frac{\partial u_{2}}{\partial \theta}-\frac{v_{2} \operatorname{cosec} c^{2} \theta}{r^{2}}\right)
\end{aligned}
$$

Assuming the continuity of velocity, stress and the jump at the surface of porous shell the boundary conditions are given as (Merikh and Mohammad 2002, Ochao-Tapia and Whitaker 1998, Alazmi and Vafai 2001)

$$
\begin{aligned}
u_{2} & =v_{2}=0, \quad \text { at } r=1 \\
u_{1} & =u_{2}, \quad \text { at } r=q=b / a \\
v_{1} & =v_{2}, \quad \text { at } r=q=b / a \\
\tau_{r \theta(1)} & =\tau_{r \theta(2)}-\frac{\beta a}{\sqrt{k}} v_{2}, \quad \text { at } r=q=b / a
\end{aligned}
$$




$$
\begin{aligned}
\tau_{r r(1)} & =\tau_{r r(2)}, \quad \text { at } \quad r=q=b / a \\
u_{1} & \rightarrow \cos \theta, \quad v_{1} \rightarrow-\sin \theta \text { as } r \rightarrow \infty
\end{aligned}
$$

where $\beta$ is a dimensionless parameter of order one known as stress jump coefficient and it's value is between -1 and 1 . Dimensionless shear and normal stress are given as

$$
\begin{gathered}
\tau_{r \theta(i)}=\frac{1}{r} \frac{\partial u_{i}}{\partial \theta}+\frac{\partial v_{i}}{\partial r}-\frac{v_{i}}{r} \\
\tau_{r r(i)}=-p_{i}+2 \frac{\partial u_{i}}{\partial r}
\end{gathered}
$$

\section{Solution of the Problem}

For axis symmetric flow there exists Stokes Stream function $\Psi_{i}$ which satisfies equation of continuity and gives

$$
u_{i}=\frac{1}{r^{2} \sin \theta} \frac{\partial \Psi_{i}}{\partial \theta}, v_{i}=-\frac{1}{r \sin \theta} \frac{\partial \Psi_{i}}{\partial r}, i=1,2
$$

Here $\Psi_{1}$ and $\Psi_{2}$ belong to region-I and region-II respectively. Eliminating pressure $p_{i}$ between equations (08) - (11) and using equation (15) we get;

$$
\begin{gathered}
E^{4} \Psi_{1}=0 \\
E^{4} \Psi_{2}-\frac{a^{2}}{k(r)}\left(E^{2}-\frac{1}{k(r)} \frac{\partial k}{\partial r} \frac{\partial}{\partial r}\right) \Psi_{2}=0
\end{gathered}
$$

Here $E^{2}$ denotes the Stokes stream function operator given by

$$
E^{2}=\frac{\partial^{2}}{\partial r^{2}}+\frac{\sin \theta}{r^{2}} \frac{\partial}{\partial \theta}\left(\frac{1}{\sin \theta} \frac{\partial}{\partial \theta}\right)
$$

Assuming permeability variation $k(r)=k_{0} r^{2}$ equation (17) becomes

$$
E^{4} \Psi_{2}-\frac{\sigma^{2}}{r^{2}}\left(E^{2}-\frac{2}{r} \frac{\partial}{\partial r}\right) \Psi_{2}=0
$$

where $\sigma^{2}=\frac{a^{2}}{k_{0}}$ is permeability parameter. Last two boundary conditions suggest that $\Psi_{i}$ should be in the form ;

$$
\Psi_{1}(r, \theta)=f_{1}(r) \sin ^{2} \theta \quad \text { for } \quad r \geq q
$$




$$
\Psi_{2}(r, \theta)=f_{2}(r) \sin ^{2} \theta \quad \text { for } \quad 1 \leq r \leq q
$$

substituting $\Psi_{i}$ in equation (16) and (19) we get

$$
\begin{gathered}
r^{4} f_{1}^{i v}-4 r^{2} f_{1}^{\prime \prime}+8 r f_{1}^{\prime}-8 f_{1}=0 \\
\left(r^{4} f_{2}^{i v}-4 r^{2} f_{2}^{\prime \prime}+8 r f_{2}^{\prime}-8 f_{2}\right)-\sigma^{2}\left(r^{2} f_{2}^{\prime \prime}-2 r f_{2}^{\prime}-2 f_{2}\right)=0
\end{gathered}
$$

Solution of the equations (22) and (23) can be written as ;

$$
\begin{aligned}
f_{1}(r)= & \frac{A_{1}}{r}+B_{1} r+C_{1} r^{2}+D_{1} r^{4} \\
f_{2}(r)= & A_{2} r^{\frac{1}{2}\left(3-\sqrt{13+2 \sigma^{2}-2} \overline{36-4 \sigma^{2}+\sigma^{4}}\right.} \\
& +B_{2} r^{\frac{1}{2}\left(3+\sqrt{13+2 \sigma^{2}-2} \sqrt{36-4 \sigma^{2}+\sigma^{4}}\right.} \\
& +C_{2} r^{\frac{1}{2}\left(3-\sqrt{13+2 \sigma^{2}+2} \overline{\frac{1}{36-4 \sigma^{2}+\sigma^{4}}}\right.} \\
& +D_{2} r^{\frac{1}{2}\left(3+\sqrt{13+2 \sigma^{2}+2} \sqrt{36-4 \sigma^{2}+\sigma^{4}}\right.}
\end{aligned}
$$

where $A_{j}, B_{j}, C_{j}$ and $D_{j}$ are constants of integration. With the above expressions for $f_{1}(r)$ and $f_{2}(r)$ equations $(20)$ and (21) represents stream function in the region I and II respectively. Using the last boundary condition we obtained $C_{1}=\frac{1}{2}$ and $D_{1}=0$. Substituting these values in equation (24) we get

$$
f_{1}(r)=\frac{A_{1}}{r}+B_{1} r+\frac{1}{2} r^{2}
$$

Now to determine the constants $A_{1}, B_{1}, A_{2}, B_{2}, C_{2}$ and $D_{2}$ appearing in equations (25) and (26) we use boundary conditions (12) which in terms of $f_{1}(r)$ and $f_{2}(r)$ can be written as

$$
\begin{aligned}
f_{2}(1) & =0 \\
f_{2}^{\prime}(1) & =0 \\
f_{1}(q) & =f_{2}(q) \\
f_{1}^{\prime}(q) & =f_{2}^{\prime}(q) \\
f_{1}^{\prime \prime}(q) & =f_{2}^{\prime \prime}(q)-\beta \sigma f_{2}^{\prime}(q) \\
f_{1}^{\prime \prime \prime}(q) & =f_{2}^{\prime \prime \prime}(q)-\sigma^{2} f_{2}^{\prime}(q)
\end{aligned}
$$

Using continuity conditions (27), we get

$$
A_{1}=\frac{1}{2}\left[A_{2}(1-a) q^{a+1}+B_{2}(1-b) q^{b+1}+C_{2}(1-c) q^{c+1}\right.
$$




$$
\begin{aligned}
& \left.+D_{2}(1-d) q^{d+1}+\frac{q^{3}}{2}\right] \\
B_{1}= & \frac{1}{2}\left[A_{2}(1+a) q^{a-1}+B_{2}(1+b) q^{b-1}+C_{2}(1+c) q^{c-1}\right. \\
& \left.+D_{2}(1+d) q^{d-1}-\frac{3 q^{3}}{2}\right] \\
A_{2}= & \frac{3 q^{3}(\beta+\sigma q)(d-c) Q}{2 \beta(P S-Q R)} \\
B_{2}= & \frac{3 q^{3}(\beta+\sigma q)(c-d) P}{2 \beta(P S-Q R)}, \\
C_{2}= & \frac{(d-a) A_{2}+(d-b) B_{2}}{(c-d)} \\
D_{2}= & -\left(A_{2}+B_{2}+C_{2}\right)
\end{aligned}
$$

Where

$$
\begin{aligned}
& P=(d-c)\left\{d\left(a^{2}-1\right)\left(a-\frac{\sigma}{\beta} q-3\right) q^{a+1}-a\left(d^{2}-1\right)\right. \\
& \left.\left(d-\frac{\sigma}{\beta} q-3\right) q^{d+1}\right\}-(d-a)\left\{\left(c^{2}-1\right)\left(c-\frac{\sigma}{\beta} q-3\right) d q^{c+1}\right. \\
& \left.-\left(d^{2}-1\right)\left(d-\frac{\sigma}{\beta} q-3\right) c q^{d+1}\right\} \\
& Q=(d-c)\left\{d\left(b^{2}-1\right)\left(b-\frac{\sigma}{\beta} q-3\right) q^{b+1}-b\left(d^{2}-1\right)\right. \\
& \left.\left(d-\frac{\sigma}{\beta} q-3\right) q^{d+1}\right\}-(d-b)\left\{\left(c^{2}-1\right)\left(c-\frac{\sigma}{\beta} q-3\right) d q^{c+1}\right. \\
& \left.-\left(d^{2}-1\right)\left(d-\frac{\sigma}{\beta} q-3\right) c q^{d+1}\right\} \\
& R=(d-c)\left\{\left(a^{2}-1\right)\left(a-\frac{\sigma}{\beta} q-3\right) q^{a+1}-\left(d^{2}-1\right)\right. \\
& \left.\left(d-\frac{\sigma}{\beta} q-3\right) q^{d+1}\right\}-(d-a)\left\{\left(c^{2}-1\right)\left(c-\frac{\sigma}{\beta} q-3\right) q^{c+1}\right. \\
& \left.-\left(d^{2}-1\right)\left(d-\frac{\sigma}{\beta} q-3\right) q^{d+1}\right\} \\
& S=(d-c)\left\{\left(b^{2}-1\right)\left(b-\frac{\sigma}{\beta} q-3\right) q^{b+1}-\left(d^{2}-1\right)\right. \\
& \left.\left(d-\frac{\sigma}{\beta} q-3\right) q^{d+1}\right\}-(d-b)\left\{\left(c^{2}-1\right)\left(c-\frac{\sigma}{\beta} q-3\right) q^{c+1}\right. \\
& \left.-\left(d^{2}-1\right)\left(d-\frac{\sigma}{\beta} q-3\right) q^{d+1}\right\}
\end{aligned}
$$


and

$$
\begin{aligned}
a & =\frac{1}{2}\left(3-\sqrt{13+2 \sigma^{2}-2 \sqrt{36-4 \sigma^{2}+\sigma^{4}}}\right. \\
b & =\frac{1}{2}\left(3+\sqrt{13+2 \sigma^{2}-2 \sqrt{36-4 \sigma^{2}+\sigma^{4}}}\right. \\
c & =\frac{1}{2}\left(3-\sqrt{13+2 \sigma^{2}+2 \sqrt{36-4 \sigma^{2}+\sigma^{4}}}\right. \\
d & =\frac{1}{2}\left(3+\sqrt{13+2 \sigma^{2}+2 \sqrt{36-4 \sigma^{2}+\sigma^{4}}}\right.
\end{aligned}
$$

So that the solution of equations in region I and region II can be given as

$$
\begin{gathered}
\Psi_{1}=\left(\frac{A_{1}}{r}+B_{1} r+\frac{1}{2} r^{2}\right) \sin ^{2} \theta ; \quad r \geq q \\
\Psi_{2}=\left(A_{2} r^{a}+B_{2} r^{b}+C_{2} r^{c}+D_{2} r^{d}\right) \sin ^{2} \theta ; \quad 1 \leq r \leq q .
\end{gathered}
$$

Using equation (15) velocity of the fluid in the increasing direction of $r$ and $\theta$ in region $\mathrm{I}$ is given by

$$
\begin{array}{r}
u_{1}=\frac{2 \cos \theta}{r^{2}}\left(\frac{A_{1}}{r}+B_{1} r+\frac{1}{2} r^{2}\right) \\
v_{1}=-\frac{\sin \theta}{r}\left(-\frac{A_{1}}{r^{2}}+B_{1}+r\right)
\end{array}
$$

and corresponding velocity in the region II is given by

$$
\begin{aligned}
& u_{2}=\frac{2 \cos \theta}{r^{2}}\left(A_{2} r^{a}+B_{2} r^{b}+C_{2} r^{c}+D_{2} r^{d}\right) \\
& v_{2}=-\frac{\sin \theta}{r}\left(A_{2} a r^{a-1}+B_{2} b r^{b-1}+C_{2} c r^{c-1}+D_{2} d r^{d-1}\right)
\end{aligned}
$$

where $A_{1}, B_{1}, A_{2}, B_{2}, C_{2}, D_{2}$ are given by equation (28) and (29) $a, b, c, d$ are given by equation (30).

\subsection{Pressure}

Using $u_{1}$ and $v_{1}$ from equation (33) in equation (8) and integrating the resulting equations we get the pressure $p_{1}$ outside the sphere as

$$
p_{1}=\frac{2 B_{1} \cos \theta}{r^{2}}
$$




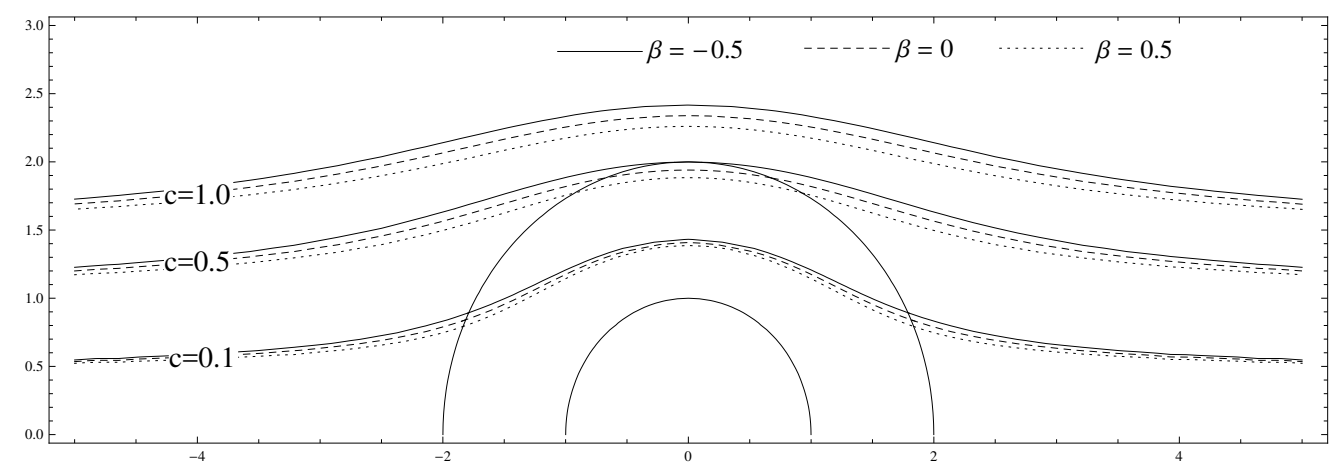

Fig. 2: Stream lines $\Psi=c(c=0.1,0.5$ and 1.0) for different $\beta$ when $\sigma=0.5$ and $q=2$.

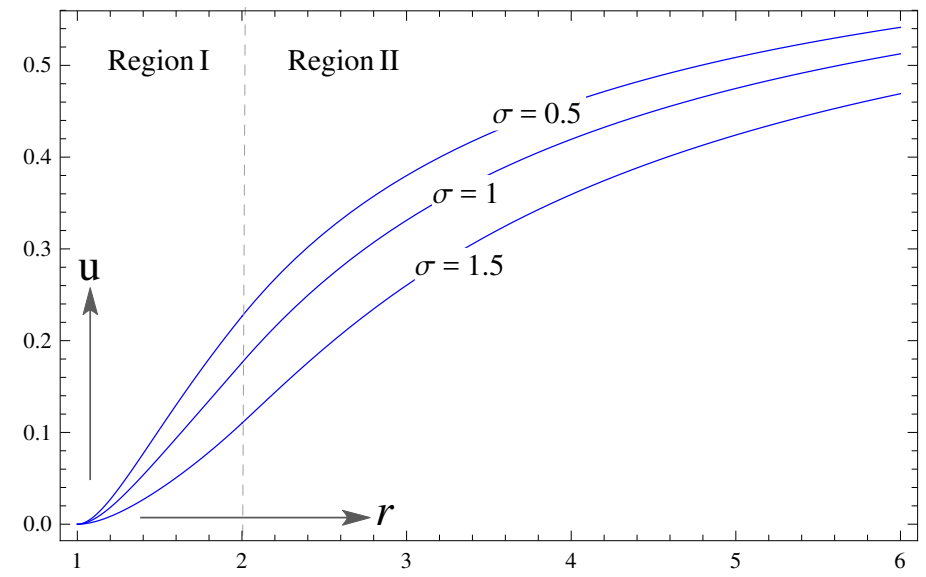

Fig. 3: Variation of radial velocity with radial distance $r$ for different $\sigma$ when $\beta=0.5, q=2$ and $\theta=\pi / 4$. 


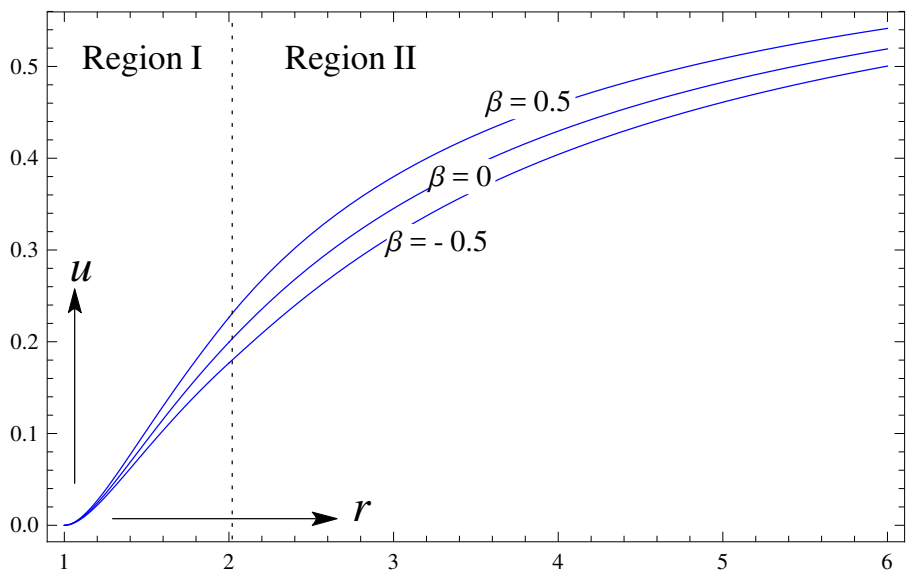

Fig. 4: Variation of radial velocity with radial distance $r$ for different $\beta$ when $\sigma=0.5, q=2$ and $\theta=\pi / 4$.

Similarly, the pressure $p_{2}$ within the porous sphere is obtained by using $u_{2}$ and $v_{2}$ from equation (34) in equation (10) as

$$
\begin{aligned}
p_{2}= & 2 \cos \theta\left[\frac{\left(\sigma^{2}-a^{2}+a+2\right)}{(3-a)} r^{a-3} A_{2}+\frac{\left(\sigma^{2}-b^{2}+b+2\right)}{(3-b)} r^{b-3} B_{2}+\right. \\
& \left.\frac{\left(\sigma^{2}-c^{2}+c+2\right)}{(3-c)} r^{c-3} C_{2}+\frac{\left(\sigma^{2}-d^{2}+d+2\right)}{(3-d)} r^{d-3} D_{2}\right]
\end{aligned}
$$

\subsection{Drag Force on the Porous Shell}

The non dimensional drag force on the surface of the porous shell of radius $q$ is given by

$$
D=2 \pi \int_{0}^{2 \pi}\left[\tau_{r r}(r) \cos \theta-\tau_{r \theta}(r) \sin \theta\right]_{r=q} \sin \theta d \theta
$$

substituting $\tau_{r r(1)}$ and $\tau_{r \theta(1)}$ from equations (13) and (14) in the above equation, we get

$$
D=24 \pi \int_{0}^{\frac{\pi}{2}}\left[-\left(\frac{2 A_{1}}{q^{4}}+\frac{B_{1}}{q^{2}}\right) \cos ^{2} \theta \sin \theta+\frac{A_{1}}{q^{4}} \sin ^{3} \theta\right] d \theta
$$

After integration, we get

$$
D=-\frac{8 \pi B_{1}}{q^{2}}
$$


In the limiting case when $\sigma \rightarrow \infty$ (i.e. when permeability of the porous shell tends to zero) for $\beta=0, \operatorname{drag} D \rightarrow 6 \pi / \mathrm{q}$. This is the well known result that the non dimensional drag force on the impervious sphere of non dimensional radius $q=\frac{b}{a}$ is $6 \pi / \mathrm{q}$.

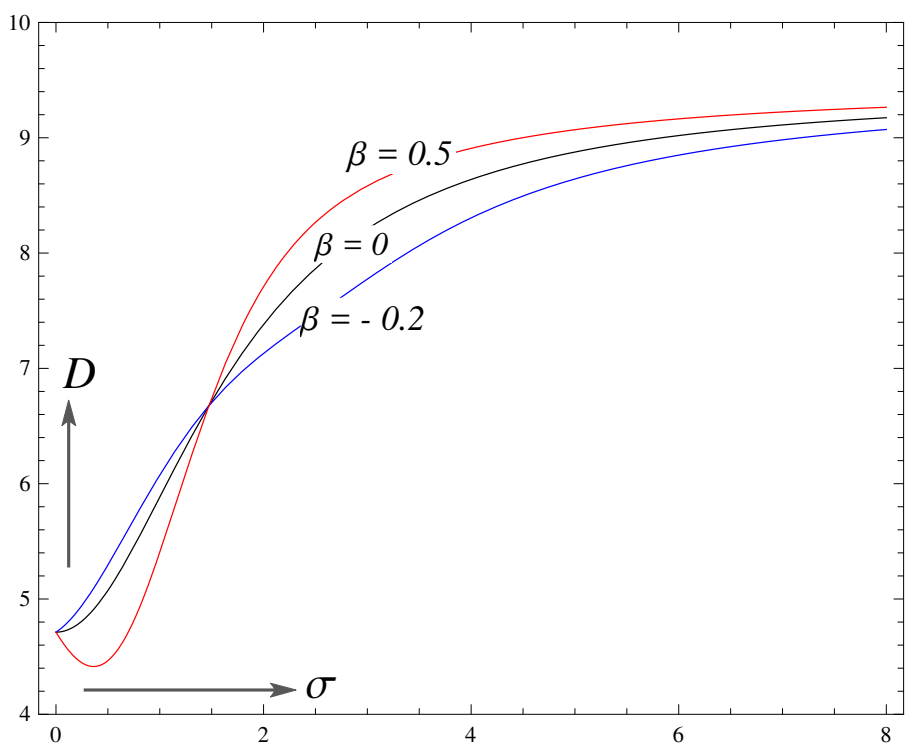

Fig. 5: Variation of Drag Force $D$ with permeability parameter $\sigma$ for different $\beta$ when $q=2$.

\section{Discussion}

Fig. (2) represents stream lines $\Psi=0.1, \Psi=0.5$ and $\Psi=1$ of the flow past the porous spherical shell. These streams are sketched for different $\beta=-0.5,0,0.5$ when $\sigma=0.5$ and $q=2$. Fig. 2 shows that there is a remarkable effect of the stress jump coefficient on the stream lines of the flow. We observe that as $\beta$ increases from the value -0.5 to 0.5 fluid flow increases through the spherical porous shell.

Fig (3) shows the variation of radial fluid velocity with radial distance $r$ for different values of permeability parameter $\alpha$, when $\beta$ and gap parameter $q$ has fixed value 0.5 and 2 , respectively. We can observe that velocity increases from the zero value (at the solid core) to the fixed value of uniform stream at large distance from the centre of spherical shell for all values of $\alpha$. Also we see 
that as permeability parameter $\alpha$ increases velocity decreases. This is because of the fact that increase in $\alpha=a^{2} / k_{o}$ means decrease in the permeability of porous shell.

Fig.(4) shows the variation of radial fluid velocity with radial distance $r$ for different values of stress jump coefficient $\beta$, when $\alpha$ and gap parameter $q$ has fixed value 0.5 and 2 , respectively. We can observe that velocity increases from the zero value (at the solid core) to the fixed value of uniform stream at large distance from the centre of spherical shell for all values of $\beta$. Also we see that as stress jump coefficient $\beta$ increases velocity increases. This effect of stress jump coefficient on the velocity is also in agreement with the fiq. 2 .

Fig.(4) shows the variation of drag force on the porous shell with permeability parameter $\alpha$ for $\beta=-0.2,0,0.5$. We observe that drag force $F$ increases with permeability parameter $\alpha$ and as $\alpha \rightarrow \infty$ drag tends to fixed value $3 \pi$ for $q=2$. Also the effect of stress jump coefficient $\beta$ on the drag force is large for small value of $\alpha$ (i.e for large permeability).

\section{Conclusion}

Steady flow of viscous fluid past a fixed porous spherical shell of radially varying permeability with solid core has been investigated. We assume that permeability of the porous shell vary according to law $k=k_{o} r^{2}$. The Brinkman and stokes equation has been used to find the analytical solution of the flow. We obtain exact expression for stream line, velocity and drag force on the spherical shell. The effect of permeability parameter and stress jump coefficient on the flow are discussed. We observe that stress jump coefficient and permeability parameter has remarkable effect on the flow hydrodynamics. The obtained results are important in applications where the porous particle is of variable permeability.

\section{References}

[1] A. A. Merrikh and A. A. Mohamad, Non-Darcy effects in buoyancy driven flows in an enclosure filled with vertically layered porous media, Int. J. Heat Mass Transf, 45, 43054313 (2002).

[2] A. Bhattacharya and G. P. Rajashekhar, Stokes flow inside a porous spherical shell:Stress jump boundary condition, ZAMP , 56, 475-496 (2005).

[3] A. Bhattacharya, Effect of momentum transfer condition at the interface of model of creeping flow past a spherical permeable aggregate, European J. Mech., 29, 285-217 (2010). 
[4] A. S. Kim and R. Yuan, hydrodynamics of an ideal aggregate with quadratically increasing permeability, J.Colloidal and Interface Ssc., 285, 627-633 (2005).

[5] A. V. Kuznetsove, Analytical investigation of Couette flow in a composite channel partially filled with a porous medium and partially filled with a clear fluid, Int.J. Heat Mass Trans., 41, 2556-2560 (1998).

[6] B. S. Padmavathi, T. Amarnath and S. D. Nigam, Stokes flow past a porous sphere using rinkman model, ZAMP, 44, 929-939 (1993).

[7] B. Barman, Flow of a Newtonian fluid past an impervious sphere embedded in a porous medium, Indian J. Pure Appl. Math., 27, 1249-1256 (1996).

[8] B. S. Bhatt, and N. C., Sacheti, Flow past a porous spherical shell using the Brinkman model, J. Phys. D: Appl. Phys., 27, 37-41 (1994).

[9] D. A. Nield and A. Bejan, Convection in Porous Media, Springer, Newyork (2006).

[10] G. P. Rajashekhar and T. Amarnath, Stokes flow inside a porous spherical shell, ZAMP, 51, 481-490 (2000).

[11] H. C. Brinkman, A calculation of the viscous force exerted by a flowing fluid on a dense swarm of particles, Appl. Sci. Res., A1, 27-34 (1990).

[12] I. P. Jones, Low Reynolds number flow past a porous spherical shell, Proc. Camb. Phil. Soc., 73, 231-238 (1973).

[13] J. H. Masliyah, G. Neale, K. Malysa and T. G. M. Van de Van, Creeping flow over a composite sphere:Solid core with porous shell, Chem.Eng.Sci., 42(2), 245-253 (1987).

[14] J. Happel and H. Brenner, Low Reynolds number hydrodynamics, Prentice Hall, (1983).

[15] J. A. Ochoa-Tapia and S. Whitaker, Momentum jump condition at the boundary between a porous medium and homogeneous fluid:Inertial effects, J.Porous Media, 1, 201-217 (1998).

[16] J. J. L. Higdon and M. Kojima, On the calculation of Stokes flow past porous particles, Int. J. Multiphase Flow, 7, 719-727 (1981).

[17] T. Grosan, A.Postelmicu and I.Pop, Brinkman flow of a viscous fluid through a spherical porous medium embedded in another porous medium, Trans. porous media, 81, 89-103 (2010).

[18] V. K. Verma and S. Datta, Analytical solution of slow flow past a heterogeneous porous sphere with radial variation of permeability using Brinkman model, J. Porous media, 5(7), 689-696 (2012)(b).

[19] Y. Qin and P. N. Kaloni, Creeping flow past a spherical shell, Z.Angew math.mech., 73, 77-84 (1993). 
